Anderseits macht aber der Unsicherheitsfaktor, mit dem jede Wetterprognose, je nach Lage in stärkerem oder schwächerem $\mathrm{Maße}$, behaftet ist, eine zu detaillierte Vorhersage unmöglich. Das Abwägen zwischen diesen beiden Faktoren ist für den Meteorologen nicht immer leicht. Zudem ist zu bedenken, daß die Wetterereignisse in ständigem Flusse sind und nicht an den festgelegten Grenzen Halt machen. Es ist daher notwendig, eine nach Regionen unterteilte Vorhersage vernünftig zu interpretieren und aus den Angaben etwas über die zeitliche Struktur des Wetterablaufes herauszulesen. Bei den meisten Fehlprognosen handelt es sich um eine zeitliche Verschiebung des Eintrittes eines bestimmten Wetterphänomens. Bei genauer Beobachtung kann in solchen Fällen der Benützer der Wetterprognose die zeitliche Korrektur oft selber anbringen.

Diese in den drei erläuterten Karten dargestellte, seit 1. Mai 1953 verwendete Einteilung hat sich unseres Erachtens bewährt. Abgesehen von einzelnen Einwendungen gegenüber gewissen Bezeichnungen (z. B. Nord- und Mittelbünden, Juranordfuß) oder Klagen über zu große Länge der Prognosen, sind bisher keine Reklamationen bei der Meteorologischen Zentralanstalt eingegangen. Es darf daraus wohl der Schluß gezogen werden, daß diese Einteilung auch bei den Benützern der Prognosen Anklang gefunden hat.

\title{
RÉSUMÉ
}

On sait que les Alpes influencent le temps d'une façon sensible en Suisse. Les caractères du temps sont souvent très différents au Sud et au Nord des Alpes. Si tel est le cas, les prévisions du temps sont respectivement différentes, de sorte qu'il est nécessaire de donner une définition précise des expressions géographiques admises dans les bulletins météorologiques. L'auteur donne les raisons sur lesquelles est fondée la nouvelle subdivision de la Suisse en zones de prévisions, telle qu'elle est utilisée quotidiemment par la Station Centrale Suisse de Météorologie de Zurich depuis le 1er mai 1953.

\section{ZUR ENTWICKLUNG DER MESSTISCHBLÄTTER DES SUNDGAUS}

\section{Franz Grenacher}

\section{Mit 2 Abbildungen}

Die folgenden Betrachtungen gelten einem Kapitel der Geschichte der Topographie und Kartographie, das zweifellos verschiedene Länder interessiert. Es handelt sich um die Meßtischblätter des Sundgaus, in dem als in einem umkämpften Grenzgebiet naturgemäß auch die Landesaufnahme wechselvolle Schicksale hatte. Sie seien hier in Kürze nachzuzeichnen versucht.

Als Sundgau wird der südlichste Teil des uns benachbarten Elsasses bezeichnet. Er umfaßte im frühen Mittelalter ein Gebiet, das vom Juranordfuß und seiner damaligen Hauptstadt Basel bis zum Eckenbach-Landgraben nördlich Gemar reicht. 1648, nach dem dreißigjährigen Krieg, schrumpfte er aber auf die Graf-, Herrschafts- und Vogtländereien von Pfirt, Altkirch, Rougemont, Belfort, Landser, Maßmünster, Sennheim und Ensisheim zusammen. Für die nachfolgenden Betrachtungen sind leicht geänderte Grenzen gezogen : Rougemont und Belfort fallen aus, da diese beiden Herrschaften 1871 bei Frankreich verblieben. Hingegen ist aus praktischen Erwägungen die Gegend von Neu-Breisach miteinbezogen. Die Kartengeschichte beginnt mit Waldseemüllers Arbeiten 1506. Sie vereinigt Basler, Elsässer, Deutsche, Österreicher und Franzosen unter ihren Kartographen. Doch stehen deren Arbeiten hier nicht zur Betrachtung.

Jahrhundertelang, bis 1871, war das Elsaß ein Bestandteil Frankreichs. Da dessen Zentralismus auch für die Kartographie gilt, gibt es seit der Besitznahme des Elsasses - mit Ausnahme einer Arbeit des Basler JAKoB MEYER - keine auf Neuvermessung beruhenden Landeskarten vom Sundgau mehr. Immer sind solche Teilstücke der Carte de France. Die letzte französische Aufnahme des Sundgaus datiert 
von 1826-1835. Die Originalblätter werden «Minutes de Levés 》genannt. Sie haben den Maßstab 1:40000 und sind nie publiziert worden. Es handelt sich um ein handgezeichnetes, inhomogenes Kartenwerk, das teils schwarz-weiß, teils farbig, teils mit Schraffen, teils mit Höhenkurven ausgeführt ist. Niemand dachte in jener Zeit an die Drucklegung und Veröffentlichung von Kartenwerken solch großen Maßstabes. Die «Minutes de Levés » dienten lediglich als Originalunterlage für die Carte de France, welche im Maßstab 1:80000 in schönen Kupferstichblättern publiziert ist. Damals war es das Beste, was für eine kartographisch führende Großmacht wie Frankreich möglich schien. Frankreich verharrte aber in der Folge bei diesem Kartenwerk. Es ist so trotz vieler Teilrevisionen überholt, muß aber dennoch für $2 / 3$ von Frankreich als beste und offizielle Karte den Dienst versehen. Viel zu langsam und viel zu spät — nach 1900 — wurden die neuen Landeskarten 1:50000 und 1:20000 in Angriff genommen. Dies war die Situation um 1871, als das Elsaß wieder an Deutschland abgetreten werden mußte. Aus der Zeit der deutschen Herrschaft kommt das Meßtischblatt.

Gleich wie bei den «Minutes de Levés 》 in Frankreich und den Siegfriedblättern in der Schweiz war das Meßtischblatt nur als Originalunterlage für die Karte von Deutschland 1:100000 gedacht. Es eignete sich nicht zur Publikation. Das heute noch übliche Meßtischblatt hat den Maßstab von 1:25000, umfaßt ein Gebiet von $120 \mathrm{~km}^{2}$ und wird in 6-18 Monaten aufgenommen. Diese Form stammt vom preuBischen Militärkartographen und General Müffling. Er ordnete, nachdem er sich für die Polyederprojektion und Gradabteilung als Grundlage für die neuen Aufnahmeblätter entschieden hatte, ab 1821 die Einführung dieser Verbesserungen zum Meßtischverfahren an. Die Gradabteilung wurde für den Kartenschnitt gewählt. Das Blatt ist begrenzt von zwei Meridianen, die beiden andern Seiten fallen mit Parallelen zusammen. Für die Terraindarstellung galt noch die Lehmann'sche Schraffenmanier. Ab 1846 wurden Höhenkurven versuchsweise eingeführt und 1852 ersetzte die Kippregel den Diopter als veraltetes Visierinstrument. Als erster ziviler Interessent verlangte der Bergbau die Veröffentlichung des ungewohnt großen Maßstabs 1:25000. 1846 wurde in einem Einzelfall, ab 1868 zögernd für weitere Grubendistrikte dem Begehren Folge geleistet. Noch 1873 war man unentschlossen, ob Höhenkurven oder Schraffen der Vorzug zu geben sei. Erst 1875 erklärte man in Deutschland Metermaß und Höhenkurvendarstellung obligatorisch. Von jenem Jahre an wurden alle Meßtischblätter publiziert, und zwar in umfangreichen Jahresleistungen. 1882 erhielten die früher lediglich mit der größten darauf befindlichen Ortschaft betitelten Blätter, eine durchlaufende Numerierung, die heute allerdings durch eine neue ersetzt ist. Heute sind für ganz Deutschland in seinen Grenzen vor 1919 die Meßtischblätter vorhanden, rund 5000 Blätter. Vielleicht war es für den Sundgau von Vorteil, daß nach einem Intervall von 50 Jahren (seit der letzten französischen Aufnahme) die Meßtischaufnahmen erst nach einer Anlaufzeit und Konsolidierung des Verfahrens in den Jahren 1879-1885 erfolgten und ab 1883 bereits die Revisionen einsetzten.

Die Sundgaukarte umfaßt ungefähr 22 Blätter. (Siehe Übersichtsblatt.) Das Werk präsentierte sich aus einem Guß, war zuverlässig und ansprechend zugleich. Es kamen Steindruckblätter in den Handel, welche auch mit von Hand, in blauer Farbe, übermalten Gewässern erhältlich waren. So standen sie, bis 1914 der Krieg ausbrach, dem Publikum als unentbehrliche und viel gebrauchte Hilfsmittel für Projekte, Verkehr und Touristik zur Verfügung. Seither aber haben sie in der Öffentlichkeit ihre Rolle ausgespielt, denn nach 1914 erfolgte ein Auslieferungsverbot der deutschen Militärbehörde. Im Jahre 1919 verlangten die siegreichen Franzosen die Auslieferung der Originalplatten, womit der weiteren Vervielfältigung ein Ende gesetzt war.

Von ihren früheren Feldzügen her gewohnt, zog 1914 die französische ebenso wie die deutsche Armee mit der Carte de France 1:80 000 resp. mit der Karte des Deutschen Reiches 1:100 000 ins Feld. Sie erachteten diesen Maßstab erfahrungsgemäß als ge- 
nügend, bis der Krieg im Schützengraben erstarrte. Die Front verlief im Sundgau beinahe geradlinig vom sog. "Largzipfel 》 zum Hartmannsweilerkopf. In dieser Situation besann sich auf beiden Seiten als erste die Artillerie auf die großmaßstäbigen

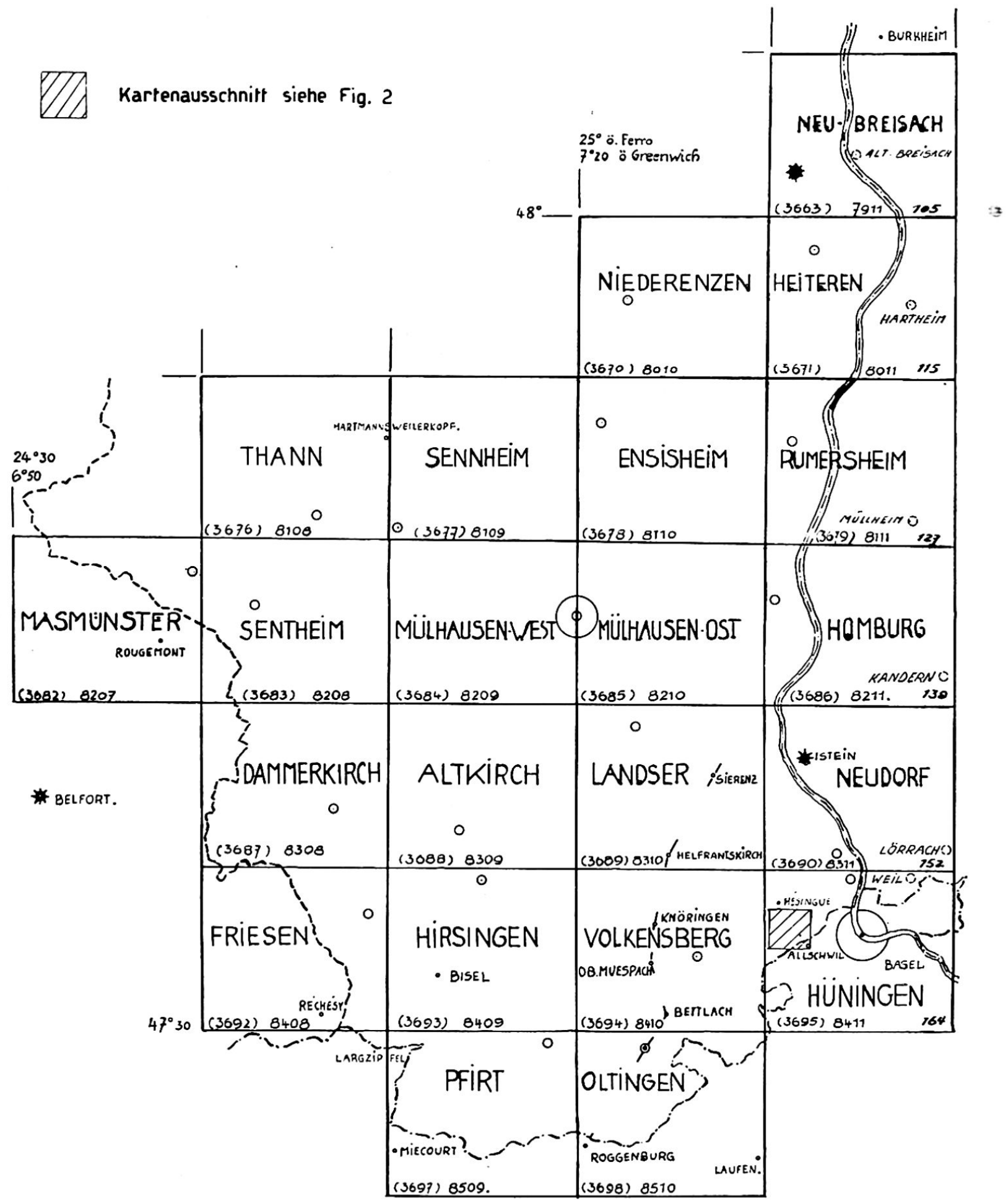

Fig. 1 Übersicht der sundgauischen Meßitischblätter:

Großgeschriebene Namen $=$ Titel der Kartenblätter. Kleine schiefliegende Namen und dreistellige Zahlen, rechtsrheinisch $=$ ehemalige Nummern und Namen der badischen Gemeinschaftsblätter. Vierstellige Zahlen in Klammern = alte, elsässischerseits bis 1939 gültige Numerierung. Vierstellige Zahlen $($ offen $)=$ seit 1939 gültige Numerierung für alle Meßtischblätter

Karten, die zum Plan-Schießen (d. h. Schießen auf nicht einsehbare Ziele) geeignet sind. Es erstaunt daher nicht, daß deutscherseits vor allem im Sundgau, wo die Front stationär geblieben war, nach den Meßtischblättern verlangt wurde. Da der Gebrauch 
der Karte 1:25000 der Truppe im Felde grundsätzlich verboten war, mußte vorerst die Heeresleitung ihre Ansicht ändern. Nachdem dies geschehen war, kam eine Serie Meßtischblätter zur Verwendung, die zuvor mit einem französischen Festungsplänen entnommenen Melde- und Schießgitter versehen worden waren.

Es handelte sich um das Soldner'sche Koordinatennetz, das als Nullpunkt das Zentrum der Festung annimmt. (SOLDNER war ein bayrischer Vermessungsfachmann, der 1809 die Cassinische Projektion verbesserte.) Drei Netze tangierten und überschnitten sich so im Sundgau: Das von Belfort, das von Istein und das von NeuBreisach. Das Netz selbst besteht aus zwei Reihen im Abstand von $4 \mathrm{~cm}$ und im rechten Winkel zueinander verlaufender und so Quadrate von ein $\mathrm{km}^{2}$ bildender feiner Linien. Am Kartenrand sorgt eine fortlaufende Numerierung für die einwandfreie Meldemöglichkeit jedes gewünschten Geländepunktes. Die bis anhin ausländischerseits weiß gelassenen Grenzblätter wurden aus den topographischen Werken der Nachbarstaaten zu Vollblättern ergänzt. Diese sogenannten Frontkartenleerdrucke begnügten sich mit Schweiz-Weiß-Druck und wurden von einer Zentralstelle geliefert. Doch bald forderte die Truppe den Aufdruck der feindlichen Stellungen. Es wurde nun für den Sundgau die Vermessungsabteilung 13 aus Württemberg aufgestellt. Sie hatte ihren Standort in Neu-Breisach, war jedoch mobil, da sie in etlichen Eisenbahnwagen untergebracht war. Aus Erkundungen, Fliegerbildern und Ergänzungen brachte sie das Erforderliche zusammen. Der von ihr bearbeitete Frontkartengraudruck zeigte eine Auswahl von trigonometrischen Punkten, die sich für die Terraineinsichtnahme speziell eignen. Sie sind mit einem Ring und fortlaufender Numerierung kenntlich gemacht (z. B. Punkt 415 Kirchturm Bisel, Blatt Hirsingen). Die vorderste eigene Linie ist lediglich mit einem blauen Strich eingetragen. In Rot sind die gegnerischen Stellungen (Schützengräben), Drahtverhaue, Laufgräben, Maskierungen, Posten usw.) gekennzeichnet. Mit einem großen roten «Geheim » betitelt, wurden sie periodisch erneuert - an die leitenden Organe der kämpfenden Truppe verteilt. (Beispielsweise trägt Blatt Hirsingen vom 4.6.1917 den Vermerk: 14. Auflage.) Wohl um leidige Verwechslungen zu vermeiden, wurde in der zweiten Hälfte des Krieges für den ganzen Sundgau nur noch das Meldegitter mit Nullpunkt der Festung Belfort beibehalten und im ganzen Kartenwerk die Numerierung danach ausgerichtet. Nebst den Stellungen des Gegners wurde auch die Eintragung der eigenen Stellungen nötig. Der Vermessungstrupp bereicherte darauf die Auflagen, die nun auf grauen Leerdruck rot die Stellungen des Gegners und in Blau diejenigen der eigenen Truppen veranschaulichten. Zusätzlich wurde noch jede Meldenummer jedes Quadrats violett eingedruckt. Damit diese höchst wichtigen Kampfmittel vertraulich und sorgfältig behandelt werden, mahnte nun der Kopfaufdruck: "Geheim, nicht in die vordersten Linien mitnehmen! »

Das Studium dieser inhaltlich erweiterten Blätter (die nun auch Feldbahnen, Batterie- und Flabstellungen, Etappenlager und Artillerieabschnittgrenzen zeigen), läßt deren tragisch-historische Rolle deutlich werden. Mit dem Kriegsende verschwinden die «Dokumente »; nur selten taucht dieses oder jenes Blatt noch auf, und es wird kaum eine Stelle geben, die sich rühmen könnte, die «Kriegsandenken 》 mehr als fragmentarisch beisammen zu haben.

Nach dem Friedensschluß ersetzten die Franzosen als neue Eigentümer der Originalplatten das Kartenwerk provisorisch durch eine mit mäßiger Sorgfalt auf 1: 20000 vergrößerte Ausgabe der selben Meßtischblätter, wobei die Toponomie (Beschriftung) deutsch blieb. Eine reguläre französische Neuaufnahme sollte diese Ausgabe möglichst bald ersetzen. Tatsächlich aber wurden diese Blätter durch die Cartes de France 1:20000 und 1:50000 ersetzt, deren Originalunterlage immer wieder das revidierte Meßtischblatt blieb. Um 1939 war von der provisorischen Ausgabe 1:20000 fast nichts mehr vorhanden. Um 1936 begann das Kartenamt der wiedererstandenen deutschen Armee eine «Sonderausgabe, nur für Dienstgebrauch» der 


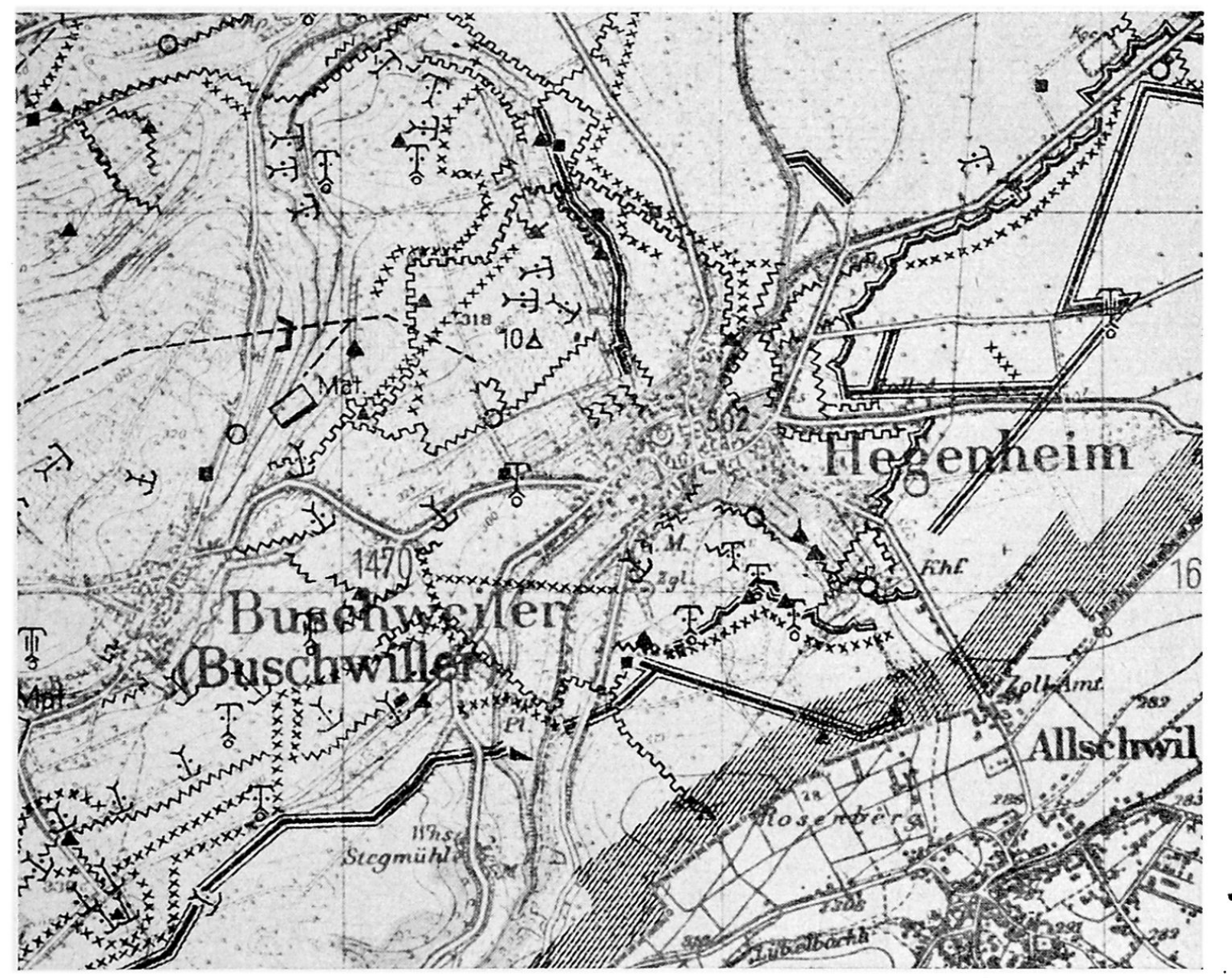

Fig. 2 Ausschnitt aus Blatt Weil (Hüningen) der Stellungskarte Frankreich 1:25000, Nr. 8411 vom 4. 4.1940

Meßtischblätter des Elsasses anzufertigen. Es ist dies ein einfacher Schwarzdruck und anfänglich, wie es scheint, aus der 1919 von den Franzosen auf 1:20 000 vergrößerten provisorischen Serie wieder auf 1:25000 verkleinert. (Eine weitere Ausgabe «A $1939 »$ von Teilen des Niederelsasses, verwandelt sogar französische Plans Directeurs 1:20000 in Meßtischblätter zurück.) Sie trägt denn auch das neue vollausgezogene Gitter «Carroyage kilometrique, projection Lambert, Zone de guerre Nord», deren Urheber der anno $1721 \mathrm{im}$ damals schweizerischen Mülhausen geborene große Mathematiker J. H. LAMBERT ist. 1938 berichtet jedoch ein Berliner Regierungsrat seinen Vermessungsbeamten vertraulich, daß die 1919 an die Franzosen geleistete Abgabe der elsässischen Originalpatten ein Schlag ins Wasser gewesen sei, da die moderne Reproduktionstechnik durch das Mittel von Umdruckplatten die Wiederherstellung aller elsässisch-lothringischen Meßtischblätter ermögliche. Im Juni lag die komplette Serie Graudrucke mit dem neuen Gaußgitter und Planzeiger «nur für den Dienstgebrauch » versehen vor.

Nach Ausbruch des Zweiten Weltkrieges war die Situation während des Drôle de guerre ähnlich dem Stellungskrieg von 1914-1918. Eine deutsche Armee lag rechtsseitig längs des Rheins einer linksseitig eingegrabenen französischen Armee gegenüber, und wiederum mußte auf deutscher Seite das Meßtischblatt als Frontkarte herhalten. Eine motorisierte Vermessungsabteilung No. 604 war diesmal für deren Ergänzung durch kriegswichtige Eintragungen zuständig. (Ihr Standort war wahrscheinlich im südlichen Schwarzwald. Vermessung, Kartographie, Felddruckerei und Kartenlager verfügten über je einen oder mehrere Lastwagen.) Wichtig waren in er- 
ster Linie die Blätter des Rheinverlaufs. Hier wurden auf badischer Seite die trigonometrischen Punkte vielfach als Standorte von Bunkern gewählt, welche Teilstücke der Siegfriedlinie bildeten. Sie wurden auf der Karte durch Ringlein mit eingezeichnetem Dreieck kenntlich gemacht. Der graue Titel heißt «Planblatt A, nur für Dienstgebrauch, als geheimer Gegenstand im Sinne von Paragraph 88 des Reichsstrafgesetzbuchs von 1934 ». Auf dem Graudruck ist der Wald in Hellgrün und die Gewässer in Blau überdruckt. Die durch Erkundungen und Fliegeraufnahmen festgestellten Positionen des französischen Gegners jenseits des Rheines sind rot miteingedruckt. Im Gegensatz zu den Blättern des Ersten Weltkrieges weisen sie eine größere Detaillierung der französischen Stellungen auf. Ein Schlüssel am Kartenrand weist 55 militärische Kartenzeichen auf: Verschiedene Bunkerarten, Panzergräben, Beobachterstände, Drahthindernisse, Minenfelder, Rodungen, Stauanlagen, Infanterieund Batteriestellungen, Flugplätze und Fernziele. Dazu werden sichere oder unsichere Objekte unterschieden. Sorgfältig sind auch die Details der Maginotlinie eingetragen. Diese beginnt in der Nähe des bernischen Roggenburg, verläuft über Oltingen, Bettlach, Obermuespach, Knöringen, Helfrantskirch nach Sierenz, wo sie in die Hartwaldung stößt und zieht dann, einen Abstand von zwei bis vier $\mathrm{km}$ vom Rhein haltend, nach Norden. Ein weiterer, roter großer Titel besagt, daß dies die «Stellungskarte Frankreichs 1:25000» sei, z. B.: «4. vorläufige Ausgabe vom 15. 4. 1940, letzter ausgewerteter Aufnahmeflugtag 1.3.1940». Die Blätter sind z. T. sehr farbenprächtig, und es ist schade, daß sie nur dem Kampf und der Zerstörung dienten. Als Mitte Juni 1940 den Deutschen bei Burkheim (Kaiserstuhl) die Rheinüberquerung und der Durchbruch der Maginotlinie gelang, müssen diese Blätter Kampfrequisiten von höchster Aktualität gewesen sein.

- Nach dem Drôle de guerre und dem Blitzkrieg folgten für den Sundgau ruhigere Zeiten, bis 1944 die Kriegswoge zum Rhein zurückschlug. Allem Anschein nach hatten in der Zwischenzeit die Deutschen die in Paris befindlichen Originalplatten wieder in Besitz genommen; denn für die Abwehrkämpfe der Schlußperiode taucht eine auf vorzüglichem Papier gedruckte, mit sehr feiner Zeichnung an die Anfänge der Meßtischblätter gemahnende Ausgabe auf. Hergestellt wurde sie vom Reichsamt für Landesaufnahme, Berlin 1944. Sie ist einfarbig, in Braun gedruckt und betitelt «Deutsche Heereskarte, nicht für die Öffentlichkeit bestimmt ». Da das eingezeichnete Gitternetz und der Planzeiger längst Allgemeingut geworden waren, erinnert sonst nichts an den Krieg, für dessen Verlauf das Blatt nur noch einen geringen Beitrag leisten konnte. Am Schluß des Zweiten Weltkrieges, im Mai 1945, wurden die nach Waltershausen verlagerten Bestände der elsässischen Meßtischblätter nebst vielen anderen Karten von den Russen erbeutet und vernichtet.

Um 1950 begann das Institut Géographique National in Paris die «Nouvelle Carte de France au 1:20 000 $\gg$ für den Sundgau in farbigen Blättern herauszugeben. Auf ihrem Blatt Altkirch No. 8 (1952, es umfaßt Blotzheim, Hésingue und Hegenheim) ist zu lesen: «Levés allemands 1885, revisées en 1935 ». Das gleiche Institut berichtet an anderer Stelle: «que ces levés ont été simplement revisés de 1932/39». Das heißt in andern Worten, daß das «Stehaufmännchen » Meßtischblatt dem Sundgau verbleibt, was es ehedem war: die Originalkarte, auf welcher alle modernen Kartenwerke fußen, außerdem aber ein getreues Spiegélbild seiner die vergangenen 84 Jahre umfassenden wechselvollen Geschichte.

\section{LITERATURNACHWEIS}

Catalogues de Cartes en Service, publiés par le Service Géographique de l'Armée. 1 1 cule : 1931 S. 12, 1936 S. 10. - BerThaud, Col.: La Carte de France, tôme II. Paris 1898, S. 48 u. folgende. - Anom.: La nouvelle carte de France, Paris 1923, S. 32. - Huguenin, M.: Historique de la Cartographie de la nouvelle Carte de France, Paris 1948, S. 61. - Anom.: La nouvelle Carte de France au 20000 e, Paris 1950, S. 33. - Walter, M.: Inhalt und Herstellung der topographischen Karte 1:25000, Gotha 1913. - Anom.: Das Reichsamt für Landesaufnahme und 


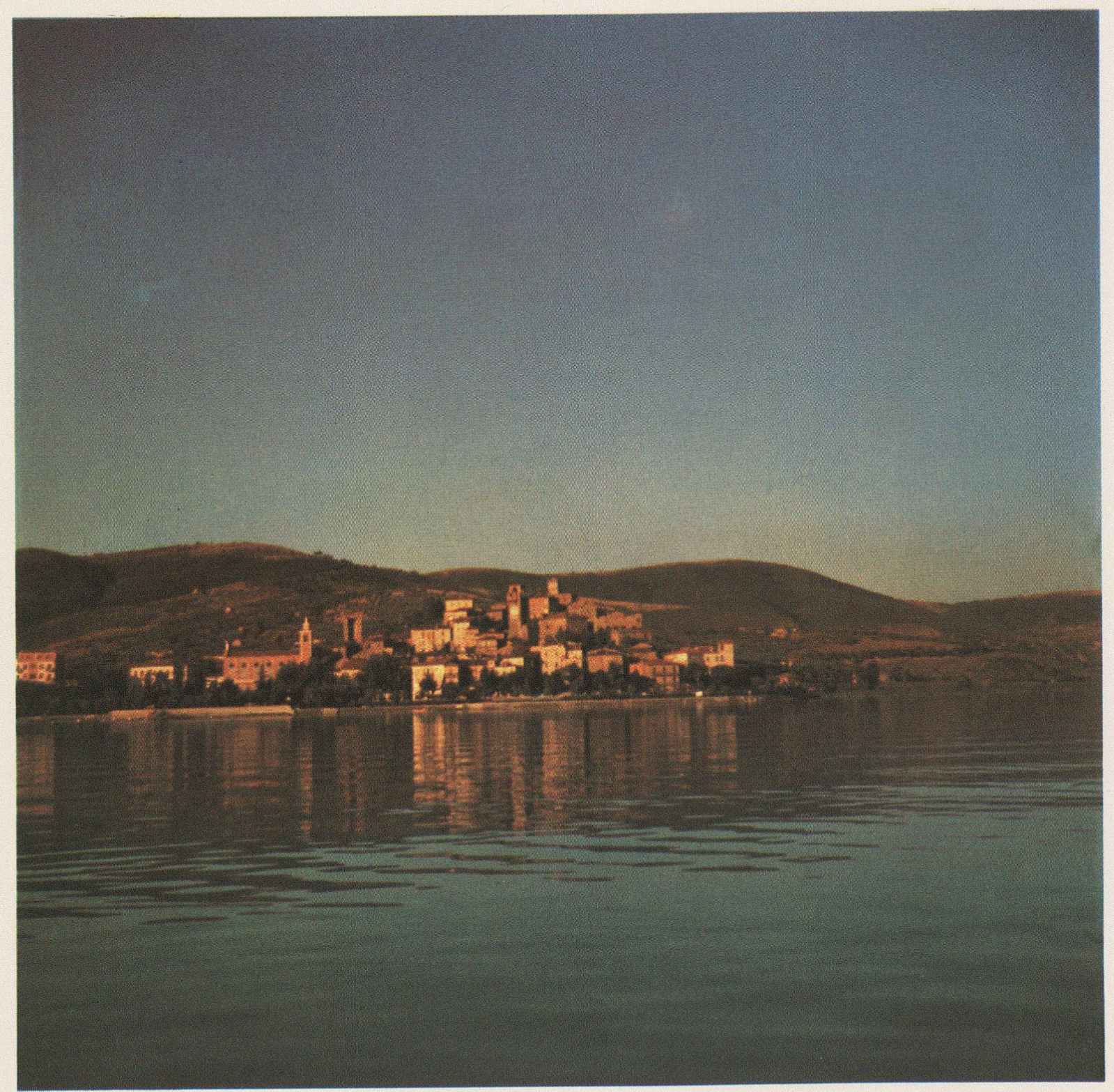

Spätnachmittag am Lago Trasimeno

Auch wer sich nicht bewußt wäre, an der Stätte einer gewaltigen historischen Entscheidung zu weilen, dürfte den Trasimeno als etwas Einmaliges, Unvergleichliches erleben, ergriffen von dieser wahrhaft idealen Landschaft. Alles an ihr ist weit, See und Hügel verschwimmen im Licht der bald scheidenden Sonne zu einer mystischen Einheit. Aus golddurchwirktem Wasserspiegel taucht feierlich das alte Passignano. 
seine Kartenwerke, Berlin 1931, S. 67. - MEYER, H.: Vorträge in maschinengeschriebenen Kopien, ungekürzte Fassung, Berlin, März 1938. 1. Die Bedeutung der Karte für Staat und Wirtschaft, S. 49. 2. Die Kartenwerke des Reichsamtes für Landesaufnahme 1:5000,1:25000, 1:100 000 und ihre Bedeutung, S. 11, 30, 41, 42, 49. 3. Die Vervielfältigung der amtlichen Kartenwerke, S. 2. Aus den Mitteilungen des Reichsamtes für Landesaufnahme, Berlin. H. DEGNER: Geschichte und Entwicklung der amtlichen preußischen Gradabteilungskarte. Jahrgang 1931, Heft 2, S. 85. von Müllek, R. aus Mitt. d. R. f. L.: Originalmeßtischblätter. Jahrgang 1935, Heft 4, S. 242.

\section{L'HISTOIRE DU «MESSTISCHBLATT»AU SUNDGAU}

Le Sundgau, c'est-à-dire la partie méridionale de l'Alsace possède des épisodes remarquables dans l'histoire de sa cartographie. Après la guerre de 1871, ayant passé de la France aux mains de la Prusse, et étant dépourvue d'une carte topographique moderne, ce pays se hâta, de 1879 à 1885, d'y étendre son système de la carte au $1: 25000 \mathrm{e}$, dont les feuilles sont tirées immédiatement de la planchette, d'ou son nom de « Messtischblatt» = feuille de la planchette. Jusqu'en 1914, ces feuilles jouissaient de l'appréciation générale et étaient utilisées couramment, mais avec le déclenchement de la guerre, la carte ne tint plus qu'un rôle purement militaire et clandestin. Ceci se continua entre les deux guerres et durant la seconde guerre mondiale jusqu'en 1945, chaque fois que les fronts militaires s'accrochaient au Sundgau. La troupe combattante, spécialement l'artillerie, considérant comme insuffisante la carte militaire normale au $1: 100000 \mathrm{e}$ réclamait le * Messtischblatt". Dans de diverses éditions on essaya différents systèmes de canevas de tir, et des groupes de cartographes militaires et d'imprimeries de campagne y enregistraient périodiquement tous les données et changements d'intérêt militaire, de telle façon que le "Messtischblatt * devint le * plan directeur" idéal de l'armée allemande au Sundgau, pour les deux guerres passées. En 1950 la France recommence à publier sa carte topographique au $1: 20000 \mathrm{e}$ en couleurs pour le Sundgau. Des notes confirment qu'elle continue de se baser sur "les levés allemands de 1885 , revisés en 1935 " - contenués dans le "Messtischblatt ", qui n’a donc pas achevé son rôle de carte originale du Sundgau.

\section{UMBRIEN}

\section{Willy Meyer}

Wenn Ferdinand Gregorovius, der wissensreiche und wanderfrohe Verfasser der «Wanderjahre in Italien », der über Umbrien herrliche Dinge gesagt hat, es an einer Stelle doch nur als «Vorstufe » zur Toskana bezeichnet, so wurde er unseres Erachtens dem schönen und in vielfacher Hinsicht reichen Lande nicht gerecht : es hat Anspruch, durchaus als etwas Eigenes gewertet $\mathrm{zu}$ werden. Wie so oft im Falle von Gegenden, die einmal ein politischer Begriff waren und heute nur noch ein landschaftlicher sind, entsprechen auch Umbriens Grenzen, so, wie man sie jetzt versteht, nicht mehr den ursprünglichen. Heute ist Umbrien der Bereich zweier italienischer Provinzen: derjenigen von Perugia, die drei Viertel von ihm, und derjenigen von Terni, die das verbleibende Viertel umfaßt. Die Gliederung ist trotzdem berechtigt; denn wenn Perugia die bedeutendste Repräsentantin altumbrisch-etruskisch-römischer und mittelalterlicher Überlieferung ist, verkörpert Terni den Gegenwartsgeist einer mächtigen Industrie. Ziffernmäßig ist die Bevölkerung Umbriens beträchtlich mehr als zur Hälfte landwirtschaftlicher Betätigung zugewandt. Umbrien verdankt seinen Namen den Umbrern, d. h. seinen ersten «historischen 》 Bewohnern, die dann von den Etruskern überwunden, mit ihnen verbündet, von den Römern besiegt und, wie sie, deren getreue Anhänger wurden. Starke Umwälzungen verursachte die Völkerwanderung, in deren Verlauf die Ostgoten viele Orte unterwarfen, viele auch zerstörten. Nachhaltiger aber war die Herrschaft der Langobarden, die in Spoleto ein Herzogtum errichteten, das sich über die umbrischen Grenzen hinaus erstreckte, und in dessen Regierung ihnen ihre Besieger, die Franken, folgten. Im hohen Mittelalter bietet Umbrien das Bild eines fortwährenden Wechsels der Gewalten: in seinen Städten lösen sich Perioden der Gemeindefreiheit mit der Tyrannis einzelner Familien oder aber Condottieri ab; innere Geschlechter- und Parteienkämpfe geben an Erbitterung und monströsen Bluttaten denen des übrigen Mittelitaliens nichts nach; die 